\title{
Alternativas para la Eliminación Doméstica de Fluor en el Agua de Consumo Humano
}

\author{
Lorenzo Valenzuela $^{(1)}$, Jorge Ramírez-Hernández ${ }^{(2)}$, Jesús A. Sol ${ }^{(1)}$ y Jaime A Reyes ${ }^{(2)}$ \\ (1) Centro de Estudios Superiores del Estado de Sonora, km 6.5 Carretera a Sonoita, \\ San Luis Río Colorado 83450, Sonora-México (e-mail: valenzuela_lorenzo@yahoo.com) \\ (2) Universidad Autónoma del Estado de Baja California, Instituto de Ingeniería, Ave. De la Normal \\ s/n Col. Insurgentes-Este Mexicali, 21280 B.C.-México (e-mail: jorger@uabc.edu.mx)
}

Recibido Jul. 14, 2010; Aceptado Ago. 16, 2010; Versión Final recibida Oct. 15, 2010

\section{Resumen}

Se evaluaron óxido de magnesio, alúmina activada, hueso granular y calcinado como métodos de eliminación de fluoruros en el agua para proponer su uso a nivel doméstico. Los pozos del área de estudio presentan concentraciones de fluoruro de 0.53-8.9 mg/l. Los resultados indican que la alúmina activada alcanza a remover el $42 \%$ en $10 \mathrm{~min}$, mientras que el hueso calcinado remueve el $43 \%$ en 5 minutos, de agua con concentraciones de fluoruro de $8.7 \mathrm{mg} / \mathrm{l}$, por lo que ambas opciones se consideran como viables. Para aumentar la remoción de fluoruro con alúmina activada es necesario variar el pH siendo más complicado su uso en el ámbito doméstico. Además, el agua tratada podría absorber aluminio de la solución de alúmina, no así con hueso calcinado. El hueso calcinado es la mejor alternativa por no producir alteraciones en las características del agua tratada y por la facilidad de obtención de este material.

\section{Alternatives for Domestic Fluoride Elimination from Household Water for Human Consumption}

\begin{abstract}
Oxide of magnesium, activated alumina, granular bone and bone chart were evaluated as methods of elimination of fluoride from water in order to propose the most feasible one for its domestic use. The water from the wells of the study area presents fluoride concentrations from 0.53 to $8.9 \mathrm{mg} / \mathrm{l}$. The results indicate that activated alumina removes $42 \%$ of fluoride in $10 \mathrm{~min}$, and the calcined bones removes $43 \%$ of fluoride in 5 minutes, from water with $8.7 \mathrm{mg} / \mathrm{l}$ of fluoride. Both methods could be considered as viable options. To increase the removal of fluoride with activated alumina is necessary to vary the $\mathrm{pH}$ being this more complicated at domestic environment. Also, the treated water could absorb aluminum from the alumina solution, which does not occur with the bone chart. The calcined bone is the best option since it does not cause alterations in the characteristics of the treated water, and this material is easy to obtain.
\end{abstract}

Keywords: fluoride, water, activated alumina, granular bone, calcined bone 


\section{INTRODUCCIÓN}

En diversas partes del mundo, es frecuente encontrar grandes zonas con aguas subterráneas que pueden aparecer con cantidades elevadas de fluoruro y aguas generalmente son explotadas para satisfacer las necesidades de consumo humano y representan un riesgo para la salud al ser ingeridas por largos periodos resultan tóxicas. Esto es debido a que el fluoruro es un elemento indispensable para la salud dental de la población, pero en altas concentraciones provoca fluorosis. (Pineda y otros 1998)

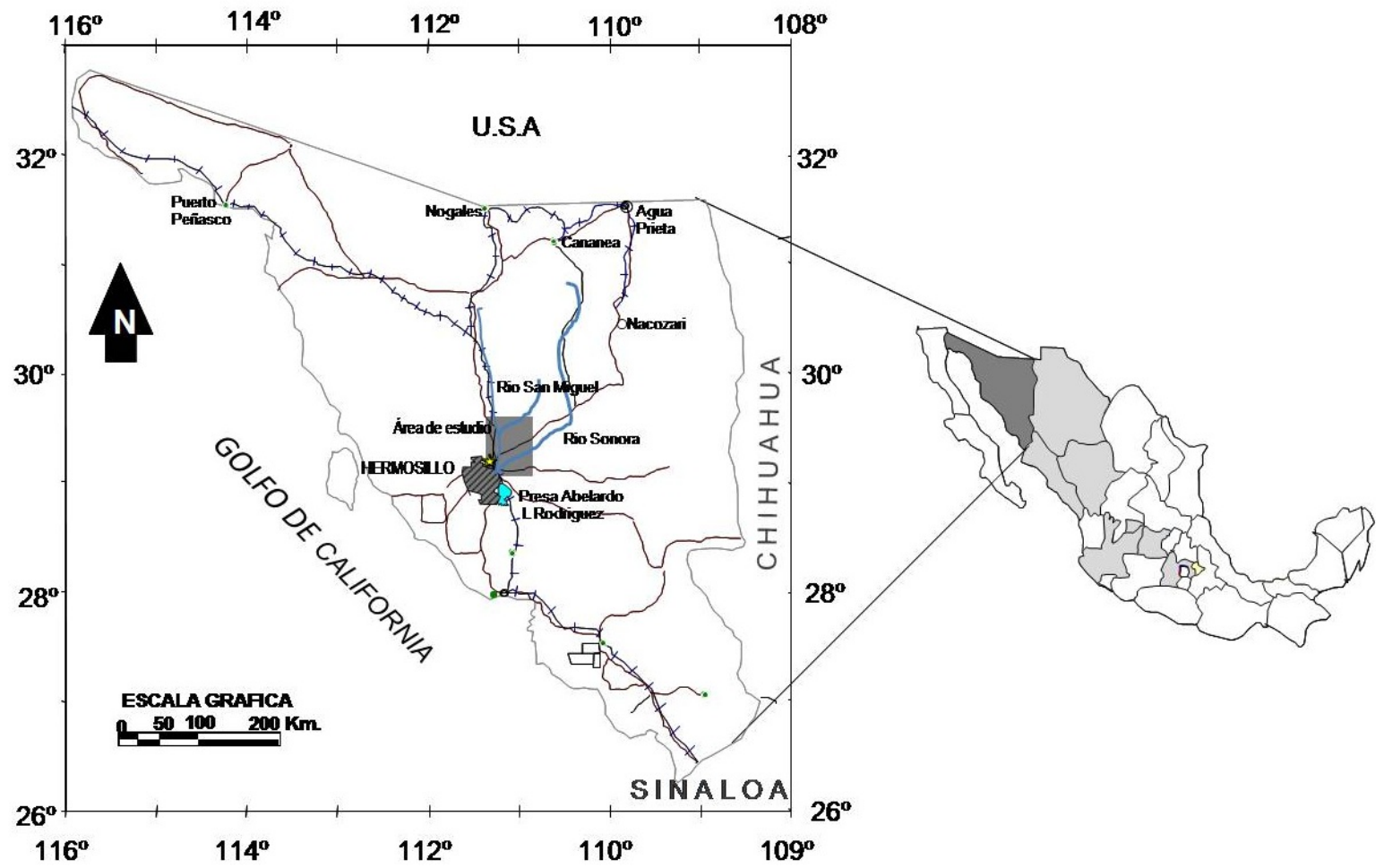

Fig. 1 Ubicación del área de estudio.

En el mundo por lo menos 25 países padecen fluorosis endémica. En la India 15 de los 32 estados se han identificado como endémicos para esta enfermedad, más de 6 millones de personas son seriamente afectadas por fluorosis y otras 62 millones están expuestas a ella (Mohapatra y otros 2004). En México aproximadamente el $6 \%$ de la población es afectada por el fluoruro contenido en el agua subterránea (UNICEF, 2004). Arriba del límite permisible se encuentra el agua en los estados de Aguascalientes, Chihuahua, Durango, Guanajuato, Estado de México, Jalisco, San Luis Potosí, Sonora y Zacatecas (Vega 2002), (figura 1). En San Luís el 70 \% de la población ha sido afectada por fluorosis dental y se prevé la aparición de fluorosis esquelética, (Gallegos y otros 2004). En Durango el $84 \%$ de los pozos que abastecen de agua a los habitantes de Guadiana excede del límite $(1.5 \mathrm{mg} / \mathrm{l})$ el $35 \%$ de la población y en áreas donde el fluoruro excede a $12 \mathrm{mg} / \mathrm{l}$, sufren daños severos, los niños en edad escolar muestran fluorosis dental, (Alarcón y otros 2001; 2002), el 33 \% de la población adulta presenta un alto grado de afección severa.

En Sonora ubicado en el Noroeste de México, (figura 1) las concentraciones detectadas en el agua subterránea, de consumo humano varía desde 0,1 hasta 7,8 mg/l. En la Ciudad de Hermosillo capital del Estado de Sonora, los pozos que abastecen la parte norte presentan la más altas concentraciones de fluoruro (Quintanar 1992). Esta ciudad, ha presentado un aumento en la demanda de agua dada la tasa de crecimiento media anual de 3,13 \%. La población ascendió a 711.512 habitantes (Gobierno Municipal 2005), siendo la ciudad más poblada del Estado.

El abasto de agua es por dos fuentes: de origen superficial, a través, de los ríos Sonora y San Miguel (figura 1) cuyos escurrimientos son almacenados en la Presa Abelardo L. Rodríguez y la de origen subterráneo obtenido de la red de pozos ubicados al Norte y Noreste de la ciudad. 
Un método para reducir la concentración de fluoruro es mezclar con aguas de pozos de más bajas concentraciones, sin embargo en la mayoría de los casos no se alcanza los niveles máximos permitidos por la Norma Oficial Mexicana NOM-127-SSAI-1994 (Diario Oficial de la Federación, 1995). Por lo tanto, es necesario aplicar métodos adicionales de eliminación de fluoruro que permitan lograr los límites apropiados de consumo.

El objetivo de este trabajo es identificar diferentes métodos de eliminación de fluoruro que sea más factible, ser usado a nivel doméstico con base en el cumplimiento de la norma NOM-127SSAI-1994 para agua potable.

\section{ANTECEDENTES}

El fluoruro es un elemento esencial para humanos y animales. La presencia de fluoruro a niveles desde 0,5 a 1,0 mg/L es benéfico para la producción y mantenimiento de huesos y dientes saludables, sin embargo una ingesta de agua con concentraciones de fluoruro $(>1,5 \mathrm{mg} / \mathrm{l})$ puede causar fluorosis ósea o dental que son enfermedades crónicas manifestadas por moteado en los dientes, ablandamientos de huesos y daños neurológicos en casos poco severos (Fan y otros 2003). Mientras que Organización Mundial de la Salud recomienda una concentración entre 0,5 a $1,2 \mathrm{mg} / \mathrm{l}$ dependiendo de las condiciones climáticas. La Norma Oficial Mexicana (NOM-127-SSAI1994) señala como concentración máxima en agua potable de $1,5 \mathrm{mg} / \mathrm{L}$.

Existen varios métodos para la eliminación de fluoruro, como precipitación, adsorción, intercambio iónico y procesos de membrana como; osmosis inversa, nanofiltración, electrodiálisis y la técnica de Nalgonda que se basan en la adición de un álcali, cloruro, sulfato de aluminio o cloruro de aluminio o ambos (Mohapatra y otros 2004). La remoción de fluoruro es factible con sulfato de aluminio pero requiere de dosis muy elevadas e indican la importancia de mantener el pH entre 6 y 7 para obtener una mejor eliminación de fluoruro. (Bischop y Sansoucy 1979). La utilización de alúm $\left(\mathrm{Al}_{2}\left(\mathrm{SO}_{4}\right)_{3} 18 \mathrm{H}_{2} \mathrm{O}\right.$ sulfato de aluminio o Alúmina activada) y óxido de calcio $(\mathrm{CaO})$, mezclados y adicionados al agua contaminada con fluoruro y con una agitación permite que se formen flóculos de hidróxido de aluminio que se remueven por simple sedimentación, esta técnica es conocida como Nalgonda.

La remoción por alúmina decrece rápidamente cuando el pH es inferior a 4.5 y superior a 8.5,la combinación de métodos para la defluorización utilizando la Técnica de Nalgonda y hueso calcinado es recomendable para la eliminación de fluoruro en el agua (Choi y Chen 1979). El carbón de hueso es la técnica más efectiva para la remoción en aguas con altas concentraciones de fluoruro, la utilización de altas concentraciones de alum para la eliminación de fluoruro no es efectiva, por el incremento de aluminio en el agua tratada (Mjengera 2000).

La capacidad de adsorción de fluoruro en hueso calcinado con mayor potencial se adsorción se obtiene cuando las partículas de hueso son pequeñas $(.5$ a $1 \mathrm{~mm})$ y la temperatura de incineración del hueso es de $500^{\circ} \mathrm{C}$ durante 2 horas (Kaseva 2006).

La hidroxiapatita, ha sido usada como implante de hueso en dientes dada la composición química y estructura cristalográfica muy similar a estos tejidos duros del humano. La remoción de fluoruro puede realizarse utilizando hidroxiapatita, calcita, cuarzo, o bien, cuarzo activado por iones férricos. Se ha reportado que el fluoruro es más rápidamente adsorbido por cuarzo, calcita y fluoruro de calcio, alcanzando el equilibrio en tan solo dos minutos en condiciones de agitación (Valenzuela y otros 2006).

El carbón activado posee la virtud de adherir o retener en su superficie uno o más componentes (átomos, moléculas, iones) del líquido que está en contacto con él. El carbón activado se denomina a cualquier clase de carbón vegetal, o de hueso calcinado que es sometido a un proceso de granulación o pulverización y que se caracteriza por poseer una superficie especifica (alrededor de 500 a $1500 \mathrm{~m}^{2}$ por gramo) (Valenzuela y otros 2006). 
Se tienen resultados favorables en la eliminación de fluoruro a bajas concentraciones con carbón de hueso, resina de intercambio, zeolitas sintéticas y alúmina activada (Hao y Huang 1986). El uso de harina de hueso desengrasado por calcinación ha sido utilizado como uno de los métodos para disminuir el contenido de flúor en el agua. (Daniele 2002).

Un método de defluoridación de bajo costo es la utilización de carbón vegetal que puede remover fluoruro de una concentración 4,10 mg/l a 1,95 en un tiempo de retención de 3 horas. Si el tiempo se incrementa a 64 horas se tiene una eliminación de hasta un $81 \%$, obteniendo la más alta retención a las 24 horas de tiempo de retención (Padmasiri y otros 1995).

La utilización de hueso como método de desfluorización fue desarrollado en Dinamarca y consiste en mezclar fosfato de sodio dihidrógenado hidratado $\left(\mathrm{NaH}_{2} \mathrm{PO}_{4}: \mathrm{H}_{2} \mathrm{O}\right)$ y cloruro de calcio $\left(\mathrm{CaCl}_{2}: 2 \mathrm{H}_{2} \mathrm{O}\right)$ en una columna de agua. La mezcla obtenida forma un lecho de contacto con el hueso calcinado que permite un flujo lento del agua con fluoruro a través de la columna, eliminando el $97.9 \%$ de fluoruro en un agua que contenía $11 \mathrm{mg} / \mathrm{l}$ con un tiempo de retención de una hora, (Dahi y otros 1996).

En una solución de fluoruro, los iones fluoruro son primeramente adsorbidos en la superficie de hidroxiapatita y el fluoruro adsorbido es intercambiado con el grupo superficial $\mathrm{OH}^{-}$más cercano de la partícula de apatita y luego intercambiado con el grupo móvil de $\mathrm{OH}$ dentro de las partículas de hidroxiapatita, resultando una muy alta absorción de fluoruro en hidroxiapatita o a partir de la reacción. (Fan y otros 2003).

$$
\mathrm{Cd}_{10}\left(\mathrm{PO}_{4}\right)_{6}(\mathrm{OH})_{2}+{ }_{n} \mathrm{~F}^{-}=\mathrm{Cd}_{10}\left(\mathrm{PO}_{4}\right)_{6}\left(\mathrm{OH}_{2-n}+\mathrm{F}_{\Pi}^{-}+{ }_{n} \mathrm{OH}^{-}\right.
$$

El óxido de magnesio puede ser utilizado como otra técnica doméstica de remoción de fluoruro, llevando a casi ebullición el agua con alto contenido de fluoruro en presencia de un exceso de óxido de magnesio. Con esta técnica es factible reducir el contenido de fluoruro en un agua de 5 $\mathrm{mg} / \mathrm{l} \mathrm{a} \mathrm{0,2} \mathrm{mg/l} \mathrm{(Trelles} \mathrm{1970).}$

\section{MATERIALES Y MÉTODOS}

Para la realización de este trabajo se utilizó la información de la empresa municipal encargada del suministro de agua potable de la ciudad denominada Agua de Hermosillo. In situ se cuantificó el $\mathrm{pH}$, la temperatura, conductividad eléctrica y en laboratorio se determinaron los iones mayoritarios y el fluoruro.

La determinación de la concentración de fluoruro en el agua de los pozos y en la solución acuosa utilizado en los métodos de remoción fue por medio del método SPADNS (APHA y otros 1992).

En este trabajo se evaluaron 4 diferentes cuatro diferentes sorbentes: óxido de magnesio, alúmina activada, hueso granular y hueso calcinado. La alúmina activada y el óxido de magnesio utilizados fueron productos grado reactivo. El hueso granular y hueso calcinado fueron preparados en laboratorio. La preparación del hueso granular consistió en hervir el hueso hasta dejarlo limpio de grasa, enseguida se dejó secar a temperatura ambiente para posteriormente triturarlo y pasarlo por malla de 60 unidades $(0.25 \mathrm{~mm})$. Para obtener el hueso calcinado éste se calcino a una temperatura mayor a los $450^{\circ} \mathrm{C}$ durante una hora, posteriormente tamizado en malla en malla 60 . La alta temperatura $\left(>400^{\circ} \mathrm{C}\right)$, la atmósfera especial del horno y el vapor generado son los factores que activan y general la porosidad. El área de superficie del carbón activado varía de 500 a 2500 metros cuadrados por gramo $\left(\mathrm{m}^{2} / \mathrm{g}\right)$ dependiendo de la materia prima y del proceso de activación (Schaefer 2003).

Los ensayos se realizaron en agua desionizada y agua de pozos de la "Captación Victoria", Se seleccionaron pozos con concentraciones entre de 2 a 9 mg/l. Para obtener concentraciones similares al agua de las muestras de pozos se le agregó fluoruro de sodio al agua desionizada. Ensayos con Alúmina Activada. Con alúmina activada se utilizaron concentraciones de 5, 10, 20 y $30 \mathrm{~g} / \mathrm{l}$ y $500 \mathrm{ml}$ de agua para determinar la concentración de fluoruro fueron tomadas muestras de 
$10 \mathrm{ml}$ de la solución en períodos de tiempo preestablecidos hasta que alcanzo el equilibrio. La concentración de alúmina de $20 \mathrm{mg} / \mathrm{l}$ fue la que mejor respuesta tuvo para los primero 25 minutos y se utilizó para evaluar el efecto del pH utilizando $\mathrm{pH}$ de 5,5, 6,5 7,5 y 8,5.

Para definir el equilibrio usando hueso granular y calcinado se trabajó con una concentración de $20 \mathrm{~g} / \mathrm{l}$. La solución y el sorbente se mantuvieron en agitación continua (60 rpm) y a temperatura de $25^{\circ} \mathrm{C}$ para tiempos comprendidos de 5 hasta $2880 \mathrm{~min}(48 \mathrm{~h})$. Al terminar cada periodo de tiempo se muestreo y se determinó la concentración de fluoruro presente. Se consideró que se alcanzaba el equilibrio cuando la concentración de fluoruro obtenida era la misma o bien que hubiera una diferencia del $5 \%$ con respecto muestra del período de tiempo anterior.

El óxido de magnesio se evaluó en concentraciones de 0,5, 1,0 y 1,5 g/l. el ensayo se realizó en muestras de medio litro, la solución se llevó a casi ebullición. Se dejó enfriar a temperatura ambiente y se tomó una muestra para evaluar la concentración de fluoruro y pH a diferentes periodos de tiempo $(0,60,180,1440$ y $2880 \mathrm{~min}$.).

\section{RESULTADOS Y DISCUSIÓN}

Los pozos de agua que abastecen de agua la parte norte de la ciudad de Hermosillo, Sonora presentan valores promedios de fluoruro que oscila de 0,53 a 7,59 mg/l, con valores más altos en fluoruro aquellos que su temperatura es mayor de $30^{\circ} \mathrm{C}$. Los pozos están localizados en un área con presencia de roca volcánica y presentaron un incremento temporal de la concentración de fluoruro. (Valenzuela y otros, 2006).

La alta concentración de fluoruro en el agua se presenta con mayor frecuencia en las aguas subterráneas donde la composición geológica del suelo y las condiciones son favorables para su disolución. Las altas concentraciones de fluoruro son asociados con los bicarbonatos $\mathrm{pH}$ y temperatura. El fluoruro tiene una correlación negativa con las concentraciones de calcio (Valenzuela y otros 2006).

Métodos de sorción. Óxido de Magnesio. En el agua del pozo La Bloquera la concentración de fluoruro se logró reducir de 8,7 mgF/l a 1,2 mgF/l con un tiempo de retención de $60 \mathrm{~min}$ (mgF/l miligramos de fluoruro en un litro de agua). Sin embargo Después de 60 minutos de tiempo de contacto aumenta la concentración de fluoruro llevándose a cabo un proceso de desorción y el pH se incrementa hasta 10 (figura 2). Este incremento de $\mathrm{pH}$ puede estar relacionado con un proceso de ablandamiento de aguas debido a la disolución de la anhidrita con una reducción de fluoruro, demostrando que esto puede ser a expensas del magnesio precipitado por la cal, alcanzando un pH de 12 donde pierde hidróxido de magnesio (Trelles 1970).

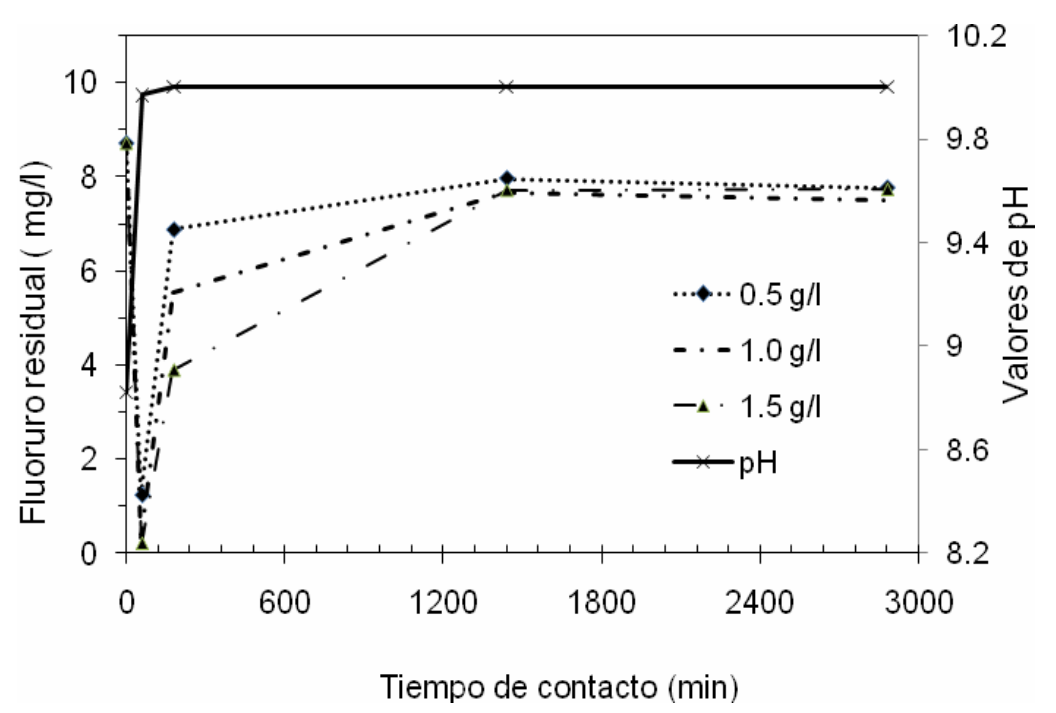

Fig. 2 Comportamiento del proceso de sorción de fluoruro y $\mathrm{pH}$ con oxido de magnesio en agua del pozo La Bloquera. 
Alúmina Activada. Para los primeros 5 minutos, el proceso de sorción usando $5 \mathrm{~g} / \mathrm{l}$ de alúmina, fue de un 1,5 mgF/l, mientras que para $20 \mathrm{~g} / \mathrm{l}$ de alúmina la porción se incrementó a 3,5 $\mathrm{mgF} / \mathrm{l}$. sin mostrar una variación significativa para 10 y $30 \mathrm{~g} / \mathrm{l}$ de alúmina. Hay una mayor velocidad de sorción en los primeros 10 minutos, con un 42\% de remoción con sorción de 3,74 mgF/L, usando $20 \mathrm{~g} / \mathrm{l}$ de alúmina activada. El equilibrio fue alcanzado con un promedio de sorción de un $47 \%$ para 10, 20 y $30 \mathrm{gr} / \mathrm{l}$ después de 60 minutos de tiempo de contacto (figura 3).

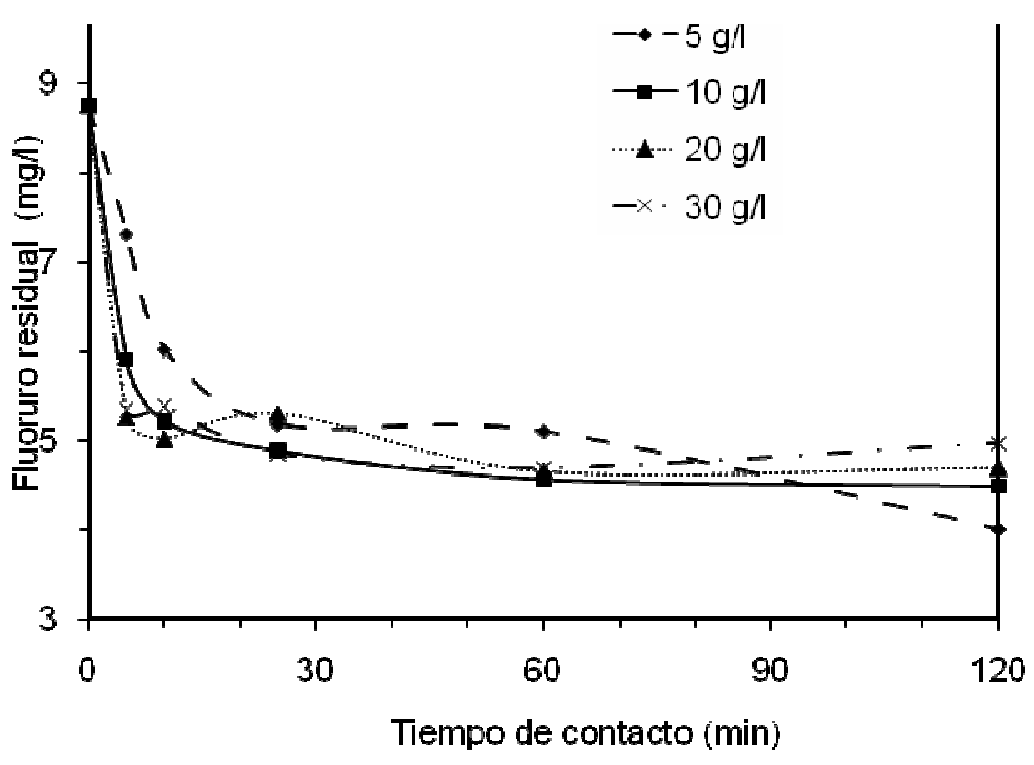

Fig. 3 Sorción de fluoruro a diferentes concentraciones de alúmina activada en agua del pozo Tronconal.

Con una baja concentración de alúmina ( $5 \mathrm{gr} / \mathrm{l})$ el equilibrio no fue alcanzado después de dos horas de tiempo de contacto. La adsorción varía desde 0,21 hasta 1,08 mgF por gramo de alúmina activada usada, varía dependiendo de la concentración inicial de fluoruro del agua tratar. Estos resultados son más bajos que los reportados por Bishop y Sansoucy (1979), con una remoción de 0,848 hasta 1,972 mg de fluoruro por gramo de alúmina. El efecto del pH fue evaluado realizando ensayos con agua de pozos y agua desionizada, la mejor respuesta fue usando $20 \mathrm{gr} / \mathrm{l}$ de alúmina activada a los primeros 5 minutos de tiempo de contacto. El pH fue cambiado utilizando ácido sulfúrico $0,01 \mathrm{~N}$ e hidróxido de sodio $0,01 \mathrm{~N}$, según el caso, para establecer valores de $\mathrm{pH}$ de, 5,5, 6,5 7,5 y 8,5. Los resultados indican que la mayor adsorción fue de $22 \%$ de remoción de fluoruro a un $\mathrm{pH}$ de 7,5 y la menor remoción, fue de 9,32\% con un pH de 6,5 para los primeros 10 minutos.

Coincidiendo con lo señalado por Wu y Nitya (1979) quiénes reportan que el intervalo de pH idóneo es de 6,0 a 7,0 cuando se usa sulfato de aluminio. Estos autores indican que se puede reducir la concentración de fluoruro 3,6 a 0,25 mgF/l usando $225 \mathrm{mg} / \mathrm{l}$ de alúmina a un pH entre 6,5 a 7,5, mientras Choi y Chen (1979) reportan que la más alta capacidad de adsorción con alúmina activada se presenta a un intervalo de pH más bajo, de 5 a 6,5.

Una tendencia a disminuir la capacidad de remoción fue observada cuando el pH fue reducido, el $\mathrm{pH}$ más bajo analizado en este trabajo fue de 5,5. El mismo efecto fue observado a pH mayores de 8,5 (figura 4). La disminución de la tasa de remoción a pH menores de 5,5 y mayores de 8,5 son explicados por Choi y Chen (1979) por la afinidad del fluoruro por los iones metálicos. Dado que los valores de $\mathrm{pH}$ del agua son mayores que 8 en la mayoría de los pozos analizados de la Captación Victoria la cantidad de remoción de fluoruro fue menor aunque el proceso fue más rápido que cuando se disminuyó el $\mathrm{pH}$ artificialmente. Cuando el $\mathrm{pH}$ se bajó se incrementó el volumen de fluoruro removido del agua natural. Para propósitos de uso doméstico se recomienda no variar el $\mathrm{pH}$, aunque el volumen de remoción de fluoruro sea menor, dado que se requieren tiempos menores para alcanzar el equilibrio y no es necesario el uso de compuestos químicos para controlar el $\mathrm{pH}$. 


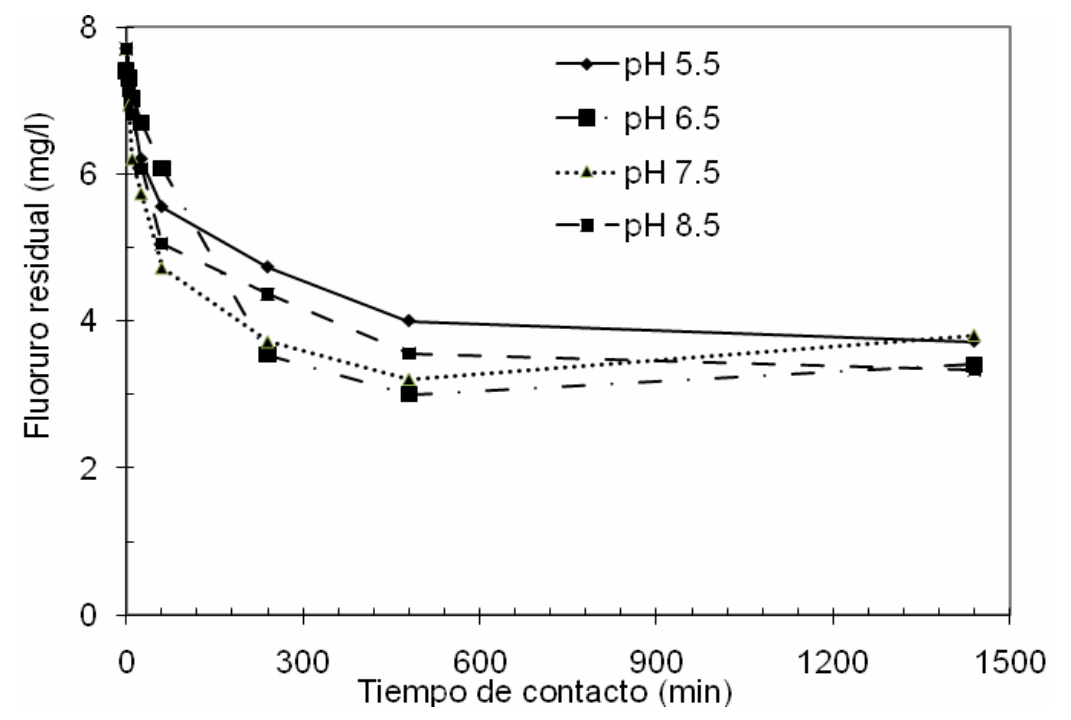

Fig. 4 Sorción de fluoruro en agua de pozo a diferentes valores de pH.

Hueso calcinado y granular. Para conocer la capacidad de sorción del hueso granular y calcinado se realizaron diferentes ensayos, tanto en agua desionizada como en agua de los pozos de "Captación Victoria".

La concentración del fluoruro residual bajo diferentes intervalos de tiempo de contacto, tanto con hueso calcinado como con hueso granular es mostrada en la figura 5. En ella se observa respuesta más rápida en la remoción de fluoruro con hueso calcinado que con hueso granular.

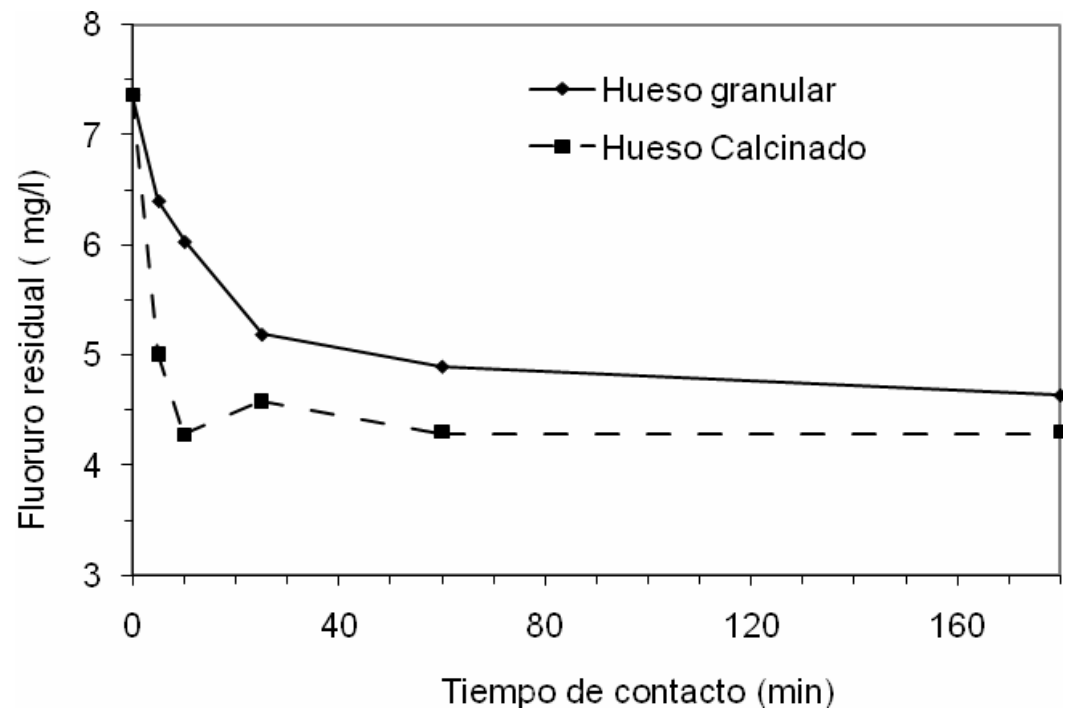

Fig.5 Comportamiento de la sorción de fluoruro utilizando $20 \mathrm{~g} / \mathrm{l}$ de hueso granular y hueso calcinado.

En 5 minutos de tiempo de contacto con carbón de hueso la adsorción de fluoruro de fue de 2,5 $\mathrm{mgF} / \mathrm{l}$, pasando de 7,36 a 5,01 mgF/l (34\%), mientras que para el mismo tiempo de contacto con hueso granular se obtuvo una remoción de 0,96 mgF/l que representa un 13\% de fluoruro removido. Estos resultados son mejores que los que reporta (Padmasiri y otros 1995) para intervalos de tiempo de contacto menores a 60 min.

Con hueso granular el fluoruro fue removido en un $18 \%(1,33 \mathrm{mg} / \mathrm{l})$, con 10 minutos de tiempo de contacto, es posible remover hasta un $37 \%$ después de 180 minutos, de tiempo sin embargo no es posible alcanzar el equilibrio para tales tiempos. 
Como se puede observar utilizando la misma cantidad de hueso granular y calcinado $(20 \mathrm{~g} / \mathrm{l})$ el hueso granular mostró una capacidad de sorción menor que el hueso calcinado (figura 6). El equilibrio no queda claramente definido a después de un intervalo de tiempo contacto de 3 horas.

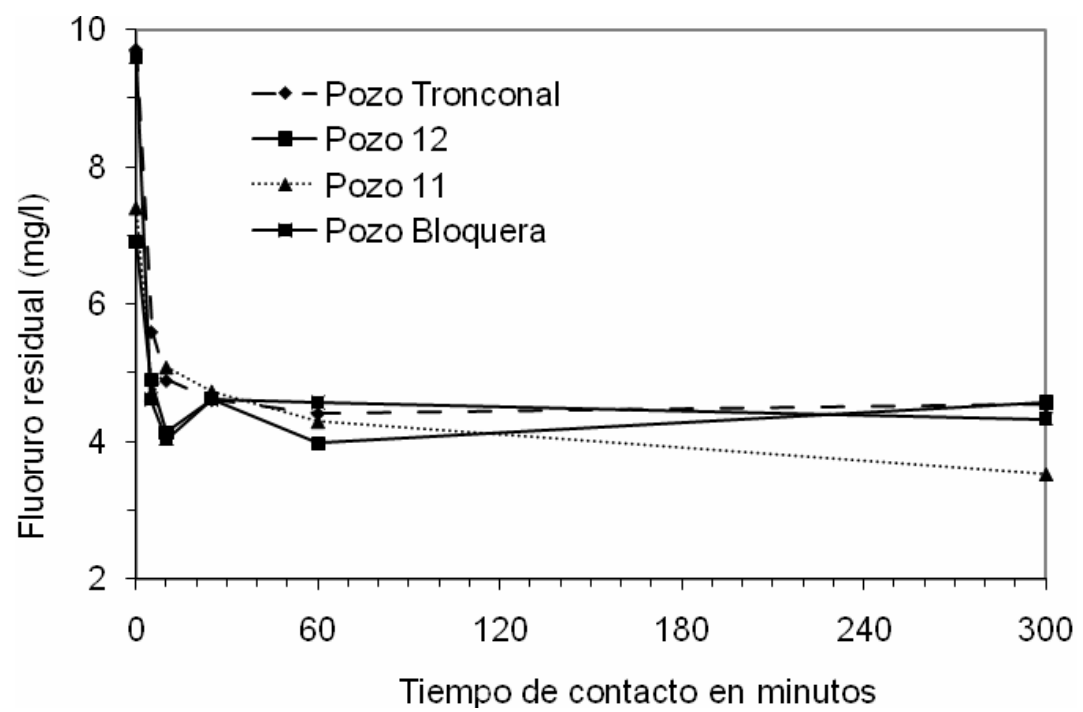

Fig. 6 Comportamiento de la sorción de fluoruro utilizando $20 \mathrm{~g} / \mathrm{l} \mathrm{de}$ hueso calcinado en cuatro pozos de La Victoria.

Al analizar algunos parámetros fisicoquímicos de las aguas antes y después del tratamiento se observó un incremento en la concentración del calcio, carbonatos, bicarbonatos y magnesio, así como un aumento en la alcalinidad, CE. En algunos casos este incremento fue del $200 \%$ mientras que el pH se elevó de 7 a 8 . El agua tratada con hueso granular mostró un olor muy desagradable por lo que no se considera como opción viable para el tratamiento de aguas para consumo humano.

Con el propósito de analizar las diferentes concentraciones del fluoruro presentes en los pozos de "Captación Victoria" y corroborar los porcentajes de remoción descritos, se realizaron ensayos en agua de 4 pozos de diferentes concentraciones (de 6,9, 7,4, 9,6, 9,7 mg/l de fluoruro), usando 20 $\mathrm{g} / \mathrm{l}$ de carbón de hueso. Los resultados indican que para un tiempo de contacto de 5 min., se obtuvo una adsorción promedio de 43,7\% (de 29 a 52\%) lo que significa una disminución de 6,9 a $4,9 \mathrm{mgF} / \mathrm{l}$ y más alta de 9,59 a 4,61 mgF/l. El equilibrio fue alcanzado antes de los 10 minutos, de tiempo de contacto con una remoción promedio de $45 \%$ de $31,26 \%$ a $57,98 \%$ esto significando disminuir de 7,39 a 5,08 y 9,59 a 5,56 mgF/l respectivamente figura 6 . Estos resultados coinciden con los reportados por (Pineda y otros 1998) alcanzando el equilibrio en menos de 10 minutos y una adsorción de 1,35 a 1,96 mgF por gramo de hueso usado para las concentraciones estudiadas.

El agua obtenida después del tratamiento con hueso calcinado mostró una coloración verdosa que fue fácilmente eliminada al hacerla pasar por carbón activado. Alteraciones de los parámetros fisicoquímicos del agua no fueron observadas por esta razón; el carbón de hueso se considera una opción viable en la remoción de fluoruro a nivel doméstico. Una alternativa para reducir la concentración de fluoruro a niveles apropiados, bajo estándares para consumo humano es la utilización de carbón de hueso calcinado.

\section{CONCLUSIONES}

El método con óxido de magnesio remueve hasta un $97 \%$ de fluoruro de la solución lo que significa bajar de 8,7 a 0,2 mgF/l, en 60 minutos de tiempo de contacto. Sin embargo después de 3 horas de tiempo de contacto se observó un elevación de pH de 8,8 a 10,1 y un incremento en la concentración de fluoruro de 3,88 $\mathrm{mgF} / \mathrm{l}$ usando $0,5 \mathrm{~g} / \mathrm{l}$ de óxido de magnesio que puede alcanzar una desorción de $90 \%$ en 48 horas de tiempo de contacto. 
Por otro lado, en el proceso con alúmina activada se puede lograr una remoción de un $42 \%$ a los 10 minutos de tiempo de contacto, lo que indica que es factible disminuir la concentración de fluoruro a niveles adecuados. Un alto porcentaje de remoción de fluoruro es obtenido variando el $\mathrm{pH}$. La máxima cantidad de fluoruro podría alcanzar un máximo (61\%) con pH de 6,5 y un tiempo de contacto de $6 \mathrm{hr}$. Esto proceso es más lento y la dificultad de hacer variaciones en el pH para que incremente la cantidad de fluoruro removido. Por otro lado, existe la posibilidad de que aumente la concentración de aluminio disuelto en la solución resultante, aunque no fue evaluado en este trabajo.

El proceso de remoción de fluoruro con hueso granular tiene tres inconvenientes; primero, se alcanza a remover el 33\% de fluoruro en una hora de tiempo de contacto, lo que lo hace un proceso lento; segundo, el agua tratada presenta un color y olor bastante desagradable que no es fácil de eliminar, y; tercero el incremento en la concentración de algunos compuestos como calcio, bicarbonato, carbonato y magnesio, así como el aumento en la alcalinidad la CE y en algunos casos el $\mathrm{pH}$.

La utilización del hueso calcinado permite obtener porcentajes de 43\% con tiempo de retención de 5 minutos alcanzando el equilibrio 10 minutos con una remoción promedio de $45 \%$, lo que indica que el proceso es más rápido y el agua obtenida no presenta alteraciones fisicoquímicas. No obstante, se observa una variación en el color que es corregido fácilmente al ser pasar el agua por carbón activado. De los procesos analizados podemos indicar que la alúmina y el hueso calcinado, son los que mejores resultados presentaron para ser usados en la eliminación de fluoruro en el agua a nivel doméstico. Sin embargo el hueso calcinado puede resultar más viable por no presentar alteraciones en las características del agua tratada, y la facilidad de obtención del material.

Se propone por lo tanto que se continúe con la práctica de mezclar aguas con alto y bajo contenido de fluoruro para reducir el contenido al menos hasta 2,0 mgF/l y el uso de hueso calcinado para reducir los niveles de fluoruro a niveles establecidos para agua de consumo humano, como una segunda etapa. Los ensayos han demostrado que esto es posible remover de 2 a $5 \mathrm{mgF} / \mathrm{l}$ en los primeros 10 minutos de tiempo de contacto. La factibilidad de obtener carbón de hueso esta segunda etapa puede realizarse en un ambiente casero y únicamente en agua de consumo humano, sin embargo es necesario realizar un estudio experimental para diseñar filtros apropiados para el proceso.

\section{AGRADECIMIENTOS}

Agradecimientos para los Ings. Rafael García Flores y Marco Antonio Alvarado de Agua de Hermosillo, al personal de Laboratorio de Química de la Universidad de Sonora, y Centro de Estudios Superiores del Estado de Sonora de. Especialmente al Dr. Michael Schorr Wienner por la revisión del documento. Esta investigación fue apoyada por el programa de SEP-PROMEP.

\section{REFERENCIAS}

Alarcón H. M. T., Martin D.I.R., Trejo V.R. y Rodríguez D., Well water fluoride, dental fluorosis and bone fractures in the Guadiana Valley Durango, Mex. Fluoride: 34, 139-149 (2001).

Alarcón, H. M. T., Martín D. A y Martín D. I. R. Concentración de flúor en el agua potable: su relación con la fluorosis dental. XXVIII Congreso Interamericano de Ingeniería Sanitaria y Ambiental Cancún. México, 27 a 31 de Octubre (2002).

APHA-AWWA-WPCF, Standard Methods for the Elimination of Water and Wastewater. Ediciones Díaz Santos S.A. p 352-364 Madrid, España (1992).

Bishop, P. L. y Sansoucy, G. Fluoride removal from drinking water by fluidized activated alumina adsorption. J Am. Water Works: 70(10):254-259 (1979).

Choi, W.W.; Chen, K.Y. The removal of fluoride water by adsorption. Water Tech: 71(10):562-570. (1979). 
Kaseva M. E. Optimization of regenerated bone char for fluoride removal in drinking water: a case study in Tanzania. Journal of Water and Health: 4(1):139-147.(2006)

Dahi, E., Mtalo, F; Njau, B. y Bregnhj, H., Defluoridation using the Nalgonda Technique in Tanzanya. $22^{\text {nd }}$ WEDC Conference, p 266-268 New Delhi, India 9 a 13 de Septiembre. (1996).

Daniele, Efecto del hueso no calcinado en la reducción del flúor en agua. Revista Científica de la Universidad de la Rioja República de Argentina. 5:3 (2) (2002).

Diario Oficial de la Federación, NOM-127-SSA1-1994, Salud ambiental, agua para uso y consumo humano-límites permisibles de calidad y tratamientos a que debe someterse el agua para su potabilización. 30 Noviembre (1995).

Fan X. Parker D.J.y Smith M.D, Adsorption kinetics of fluoride on low cost materials. Water Res: 37:4929-4937. (2003).

Gallegos G., Medellin M. P. y Passo E. M.S. Extracción de Agua Subterránea a Partir de Sistemas de Flujo Regional en San Luis Potosí: Fluoruro y Geología del Subsuelo. XXXIII Congreso IAH, $7^{\circ}$ Congreso ALHSUD. Zacatecas México, 11 a 15 de Octubre (2004).

Gobierno Municipal Disponible de http://www.hermosillo.gob.mx /transparencia/ hermocifras.html. (2005).

Hao, O. J. A.M. y Huang, C.P. Adsorption Characteristics of fluoride onto hydrous alumina. J. Env.I Eng.:112(6):1055-1069. (1986)

Mjengera $\mathrm{H}$., Defluoridation of drinking water in Tanzania. Technical Annual Report. WARFSA Swedish International Development Agency: 1-25. (2000).

Mohapatra D., Mishra D. Mishra S:P, Chaudhury G.R. y Das R.P.,Use of oxide minerals to abate fluoride from water. J of C. and Int. Sc.: 275: 355-359 India. (2004).

Padmasiri J.P., Fonseka W.S.C.A. y Liyanapatabendi T. Low cost fluoride removal by an upward fluor household filter. Water Supply 1995: 13 (3 y 4):59-64 Osaka (1995).

Pineda H, Reartes N, Sereno S. Frangie S, Bolonga C. Sereno L., Eliminación de fluoruros utilizando hydroxiapatita. XXVI Congreso Interamericano de Ingeniería Sanitaria y Ambiental. AIDIS Asociación Interamericana de Ingeniería Sanitaria y Ambiental. Córdoba Argentina, 1 a 5 de Noviembre (1998).

Quintanar, V. A. I., Niveles de flúor en el agua potable consumo humano y su relación con la salud dental del estado de Sonora. México. Tesis de Maestría. Centro de Investigación en Alimentación y Desarrollo A. C. Hermosillo, Son. (1992).

Schaefer K. Carbón Activado magia negra para tratamiento de agua. Agua Latinoamericana: 3(5):1-5. (2003).

Trelles, A. R. El problema sanitario de las aguas destinadas a la bebida humana con contenido elevado de arsénico, vanadio y flúor. Facultad de Ingeniería. Universidad de Buenos Aires, Argentina (1970).

UNICEF., Fluoride in water: an overview cited (2004).

Valenzuela V. L., Ramirez H. J., Reyes L. J., Sol U. J. A., Lázaro M. O., The origin of fluoride in groundwater supply to Hermosillo city, Sonora. Environmental Geology: 51(1):17-27. (2006).

Vega G. S., Riesgo Sanitario Ambiental por la presencia de arsénico y fluoruro en los acuíferos de México. XXVIII Congreso Interamericano de Ingeniería Sanitaria y Ambiental. Cancún. México, 27 a 31 de Octubre (2002).

Wu Y. C y Nitya, A., Water defluoridation with alumina activated. Journal the Environmental Engineer Division. 105(EE2):357-367. (1979). 